\title{
Erratum to: Informative metabolites identification by variable importance analysis based on random variable combination
}

\author{
Yong-Huan Yun ${ }^{1} \cdot$ Liang $\mathrm{Fu}^{1} \cdot$ Bai-Chuan Deng $^{1} \cdot$ Guang-Bi Lai $^{2}$. \\ Carlos M. Vicente Gonçalves ${ }^{3} \cdot$ Hong-Mei $\mathrm{Lu}^{1} \cdot$ Jun Yan $^{1} \cdot$ Xin Huang ${ }^{1}$. \\ Lun-Zhao $\mathrm{Yi}^{4} \cdot$ Yi-Zeng Liang ${ }^{1}$
}

Published online: 22 January 2016

(C) Springer Science+Business Media New York 2016

\section{Erratum to: Metabolomics (2015) 11:1539-1551 \\ DOI 10.1007/s11306-015-0803-x}

The original version of this article unfortunately contained a mistake. The family name and surname of the author Liang $\mathrm{Fu}$ was incorrect. The author name is corrected through this erratum.

The online version of the original article can be found under doi: 10.1007/s11306-015-0803-x.

Lun-Zhao Yi

ylz7910@hotmail.com

$\triangle$ Yi-Zeng Liang

yizeng_liang@263.net; yunyonghuan@foxmail.com

1 College of Chemistry and Chemical Engineering, Central South University, Changsha 410083,

People's Republic of China

2 Heilongjiang University of Chinese Medicine, Harbin 150040, Heilongjiang, People's Republic of China

3 Department of Chemistry, Faculty of Mathematics and Natural Sciences, University of Bergen, Bergen 5020, Norway

4 Yunnan Food Safety Research Institute, Kunming University of Science and Technology, Kunming 650500,

People's Republic of China 